\title{
Aspects cellulaires et moléculaires de la transduction mécano-électrique dans l'oreille interne
}

Les stimulations auditives sont perçues par les cellules ciliées de la cochlée qui les couplent à l'ouverture de canaux ioniques sans faire intervenir de messagers intracellulaires. Ces canaux pourraient être identiques aux stretch-activated channels des cellules musculaires. Ils présentent un phénomène $d$ 'adaptation dépendant du calcium, sous le contrôle, semble-t-il, du système nerveux efférent. Alors que chez les amphibiens, les reptiles et les oiseaux, ces premiers événements engendrent des oscillations électriques à la base de la sélectivité fréquentielle de la cochlée, chez les mammifères ils déclenchent des contractions rapides des cellules ciliées externes qui amplifient sélectivement le signal acoustique et le transmettent aux cellules ciliées internes qui font synapse avec les fibres nerveuses auditives.

\section{Didier Dulon Jean-Marie Aran}

\section{ADRESSE}

D. Dulon: chargé de recherche à l'Inserm. J.-M. Aran : directeur de recherche à l'Inserm. Laboratoire d'audiologie expérimentale, Inserm U. 229 et université de Bordeaux II,

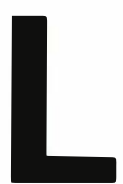

es principales étapes des réactions biochimiques qui régissent la transduction visuelle sont maintenant bien définies, avec la transformation de la rhodopsine sous l'action de l'énergie photonique et la modulation du taux de GMPc qui contrôle l'activité des canaux sodium [1]. En revanche, les réactions moléculaires intervenant dans la transduction mécano-électrique des cellules ciliées sensorielles de l'oreille interne restent encore mal connues. Cette absence de données a u niveau de la physiologie et de la biochimie des cellules ciliées est essentiellement due à la complexité de l'organisation des récepteurs et au faible nombre de cellules sensorielles composant les organes auditif et vestibulaire, qui rendent très difficiles les analyses biochimiques traditionnelles. En effet, le récepteur auditif est seulement constitué de quelques milliers de cellules (par rapport aux plusieurs millions de photorécepteurs de la rétine). De plus, ces cellules sont difficilement accessibles car elles sont enchâssées dans une coquille (la cochlée), ellemême enfouie au sein de la structure osseuse du rocher. Cependant, des techniques qui permettent $d$ 'isoler et de maintenir in vitro les organes récepteurs de l'audition ou de l'équilibre ont été développées ces dernières années. Il est donc maintenant possible d'explorer le comportement mécanique, électrique et biochimique des cellules ciliées sensorielles in vitro, dans différentes conditions de stimulation et donc d'approcher les mécanismes de base de la transduction mécano-sensorielle.

\section{La transduction mécano-électrique}

Les cellules ciliées sensorielles ont une morphologie très polarisée, avec un pôle apical, où se situe la plaque 


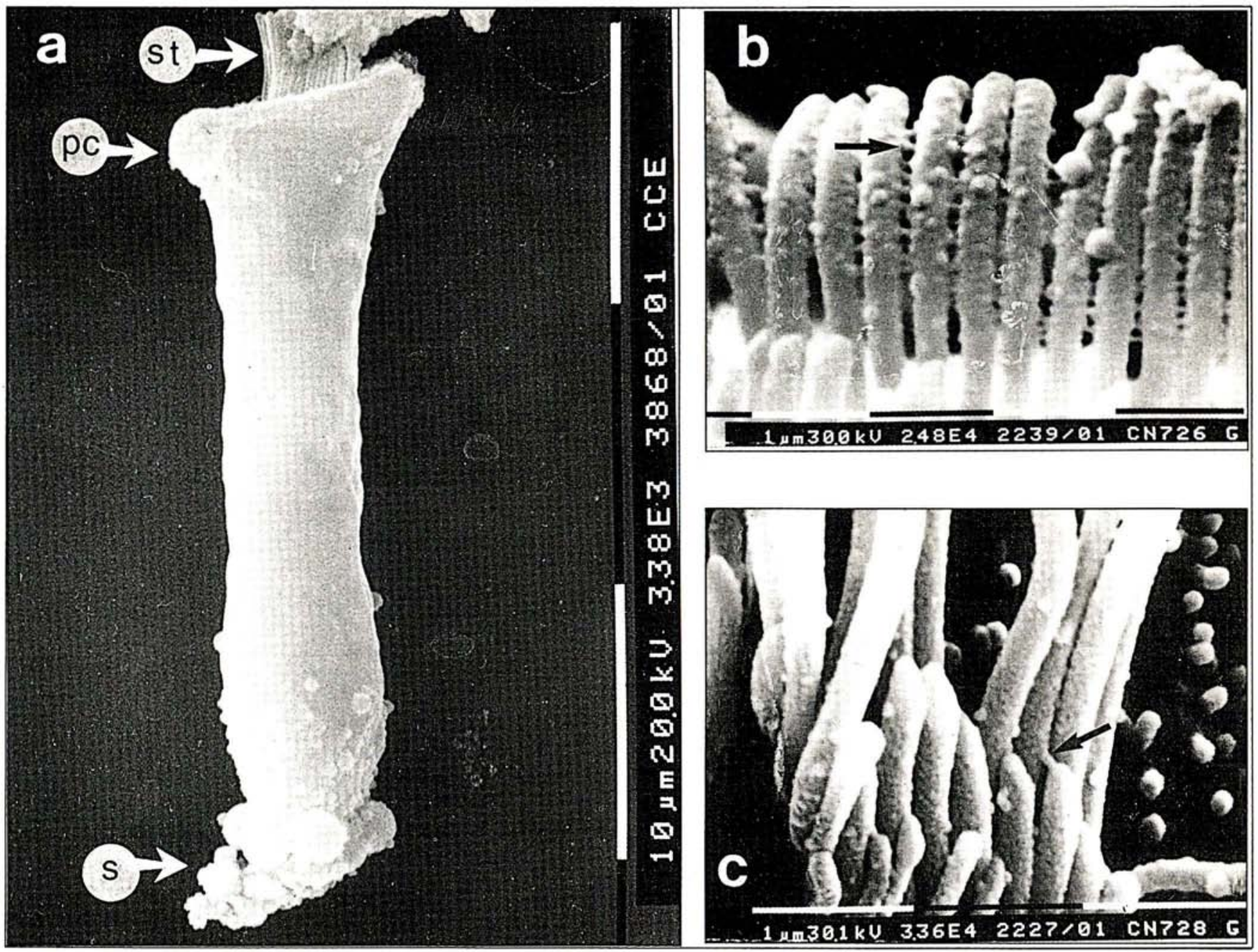

Figure 1. Morphologie d'une cellule ciliée isolée de cochlée de cobaye observée en microscopie électronique à balayage (a). st : touffe des stéréocils; pc : plaque cuticulaire; $s$ : boutons synaptiques. Au niveau de la touffe ciliaire, à plus fort grossissement, chaque stéréocil peut être individualisé. On peut observer les ponts interciliaires (flèches), les ponts médians entre cils d'une même rangée (b) et les liaisons apicales reliant le sommet des cils les plus courts aux cils de la rangée supérieure adjacente (c). Ces liaisons apicales dont la nature moléculaire reste encore inconnue seraient responsables du processus d'ouverture et fermeture des canaux de transduction selon Hudspeth [8].

cuticulaire sur laquelle est dressée la touffe ciliaire, et un pôle basal autour duquel s'organisent les synapses nerveuses (figure 1). Ces cellules sont au contact de deux milieux extracellulaires de composition ionique très différente (pour revue voir O. Sterkers et al. dans ce numéro p. 755 [2]). La touffe ciliaire est au contact de l'endolymphe, dont la composition riche en $\mathrm{K}^{+}(150 \mathrm{mM})$ et pauvre en $\mathrm{Na}+(1 \mathrm{mM})$ est proche de celle du milieu intracellulaire. Le corps cellulaire des cellules est, au contraire, au contact de la périlymphe, qui pré$\mathrm{m} / \mathrm{s} n^{\circ} 8$, vol. 6 , octobre 90 sente les concentrations classiques d'un milieu extracellulaire en cations $\mathrm{K}+(5 \mathrm{mM})$ et $\mathrm{Na}+(140 \mathrm{mM})$. Il faut également rappeler la polarisation électrique exceptionnelle de l'endolymphe dans la cochlée ( + $80 \mathrm{mV}$ environ par rapport à la périlymphe), ce qui réalise une différence de potentiel de l'ordre de $150 \mathrm{mV}$ à travers la membrane des cils et la lame cuticulaire, entre endolymphe et milieu intracellulaire (qui est à $-70 \mathrm{mV}$ environ). La touffe ciliaire, composée de quelques centaines de cils cylindriques, cons- titue véritablement le capteur des vibrations mécaniques (figure $1 b$ ). L'implantation de ces cils sur la cuticule présente une polarisation radiale latéralement au corpuscule basal, structure qui correspond à la racine d'un kinocil disparu au cours du développement dans la cochlée des mammiferes. La déflexion de la touffe ciliaire vers le corpuscule basal produit une dépolarisation excitatrice de la cellule alors que le déplacement en sens inverse produit une hyperpolarisation inhibitrice [3]. Le potentiel récepteur cellulaire peut être effecti- 


\section{RÉFÉRENCES}

1. Plouet J, Dorey C. La transduction visuelle. médecine/sciences $1987 ; 4: 192-7$.

2. Sterkers O, Ferrary E, Tran Ba Huy P. Production des liquides de l'oreille interne. médecine/sciences $1990 ; 6$ : 755-61.

3. Flock $\AA$. Sensory transduction in hair cells. In : Lowenstein WR, ed. Handbook of Sensory Physiology, vol. 1. Principles of Receptor Physiology. Berlin: Springer-Verlag, $1971 ; 396-441$

4. Sellick PM, Patuzzi R, Johnstone BM. Measurement of basilar membrane motion in the guinea pig using the Mössbauer technique. J Acoust Soc Am 1982; 72 : 131-41.

5. Tilney LG, Saunders JC. Actin filaments, stereocilia, and hair cells of the bird cochlea I. Length, number, width, and distribution of stereocilia of each hair cell are related to the position of the hair cell on the cochlea. J Cell Biol $1983 ; 86: 807-21$.

6. Tilney LG, Egelman EH, DeRosier DJ, Saunders JC. Actin filaments, stereocilia, and hair cells of the bird cochlea II. Packing of actin filaments in the stereocilia and in the cuticular plate and what happens to the organization when the stereocilia are bent. J Cell Biol 1983 ; 96 : 822-34.

7. Pickles JO, Comis SD, Osborne MP. Cross-links between stereocilia in the guinea pig organ of Corti, and their possible relation to sensory transduction. Hear Res $1984 ; 15: 103-12$.

8. Hudspeth AJ. How the ear's works work. Nature 1989 ; 341 : 397-404.

9. Ohmori H. Mechano-electrical transduction currents in isolated vestibular hair cells of the chick. J Physiol (Lond) 1985; 359 : 189-217.

10. Ohmori H. Mechanical stimulation and fura-2 fluorescence in the hair bundle of dissociated hair cells of the chick. J Physiol (Lond) 1988 ; 399: 115-37.

11. Guharay F, Sachs F. Mechanotransducer ion channels in chick skeletal muscle : the effect of extracellular $\mathrm{pH}$. $J$ Physiol (Lond) 1984 ; 352 : 685-701.

12. Sachs F. Mechanical transduction in biological systems. CRC Critical Review in Biomedical Engineering 1988; 16: 141-69.

13. Howard J, Roberts M, Hudspeth AJ. Mechanoelectrical transduction by hair cells. Ann Rev Biophys Chem 1988 ; 17 : 99-124. vement enregistré par une microélectrode intracellulaire lors de la stimulation acoustique de la cochlée in vivo ou lors de la déflexion des stéréocils d'une cellule in vitro. La réponse électrique de la cellule sensorielle du récepteur auditif est fonction de la fréquence et de l'intensité de la stimulation. A sa meilleure fréquence, la sensibilité au déplacement de la touffe ciliaire est extrême. En effet, on calcule que le seuil de réponse des cellules est atteint pour un déplacement de l'ordre de $0,3 \mathrm{~nm}$ [4]. L'endolymphe étant très riche en $\mathrm{K}^{+}$, il est fort probable que ce cation constitue l'essentiel du courant ionique de transduction au niveau de la touffe ciliaire.

Structure et organisation des stéréocils. Les stéréocils, bien décrits par Tilney et al. [5,6], sont de véritables extensions membranaires des cellules sensorielles : le cytoplasme ciliaire est en relation directe avec celui du corps cellulaire. Ces prolongements cellulaires ont une structure rigide (stéréocils). Cette rigidité est conférée par un réseau longitudinal de filaments d'actine espacés de $125 \AA$ et reliés entre eux par des ponts protéiques constitués de fimbrine. Ces filaments d'actine sont organisés en réseau hexagonal qui s'étend sur toute la longueur du cil et vient s'ancrer et s'associer à un autre réseau transversal d'actine, $\alpha$ actinine, et tropomyosine au niveau de la plaque cuticulaire. Les stéréocils présentent un diamètre uniforme de 500 à $900 \mathrm{~nm}$ à leur partie supérieure. Celui-ci diminue progressivement dans la partie basale à l'entrée de la plaque cuticulaire pour atteindre alors un diamètre inférieur à $100 \mathrm{~nm}$. Les stéréocils sont disposés en général en trois rangées successives et ont une longueur allant de $3 \mu \mathrm{m}$ pour la rangée des cils les plus courts à $7 \mu \mathrm{m}$ environ pour la rangée des cils les plus longs (figure 1b). Ils sont interconnectés par des liaisons filamenteuses (liens) dont la nature moléculaire est encore inconnue. Ces liens sont de deux types: des liens transversaux, reliant la partie médiane des cils, et des liens apicaux plus fins, qui viennent connecter le sommet du cil le plus court à la région sub-apicale du cil adjacent le plus grand (figure 1c). Cet ensemble de données morphologiques conditionne le comportement mécanique de la touffe ciliaire. Ainsi, les liens entre cils d'une même rangée renforcent la cohésion de la touffe et offrent une résistance plus grande aux mouvements de liquide, l'amincissement des cils à la base constitue un axe de rotation, l'ensemble - relativement rigide - peut aussi entraîner un pivotement de la plaque cuticulaire lors de l'application d'une force au niveau des cils. Enfin, les liens au sommet des cils, les reliant aux cils plus grands de la rangée suivante, pourraient être impliqués dans les mécanismes d'ouverture et fermeture des canaux de transduction [7].

La transduction mécano-électrique est un phénomène direct. Comment une force mécanique conduisant à la déflexion des stéréocils produit-elle un signal électrique cellulaire ?

La théorie qui prévaut à l'heure actuelle fait intervenir la notion de canaux ioniques insérés dans la membrane plasmique des stéréocils, qui voient leur conductance se modifier consécutivement à la déflexion du cil. De nombreux arguments apportés par Hudspeth [8] suggèrent que la transduction est ici un phénomène direct ne faisant pas intervenir de messagers intracellulaires comme dans le cas de la transduction photonique ou chimique. L'un des principaux arguments pour l'existence d'un phénomène de transduction couplé directement au stimulus mécanique est tiré du fait que le temps de latence de la réponse électrique cellulaire est extrêmement bref (bien inférieur à la milliseconde). Les enregistrements d'activité électrique révèlent que ces canaux constituent des pores cationiques peu sélectifs qui laissent passer des ions tels que le potassium et le calcium.

Localisation et fonctionnement des canaux de transduction. Bien qu'il n'existe aucune preuve biochimique directe, il est tentant de penser que les canaux de transduction sont organisés au niveau des cils. Les cils sont de véritables prolongements cytoplasmiques de la cellule ciliée et donc toute variation de conductance ionique se répercute instantanément sur le potentiel intracellulaire. 
Comment la déflexion des cils peutelle provoquer l'ouverture de ces canaux?

Les éléments de réponse à cette question proviennent d'expériences indirectes sur leur activité électrique. Il paraît encore difficile d'apposer de manière précise une microélectrode de patch-clamp sur les fines structures membranaires des cils, ce qui permettrait d'isoler l'activité d'un seul canal.

L'analyse, à travers des enregistrements électriques intracellulaires, du courant de transduction et du potentiel récepteur engendré par une force appliquée sur les cils permet de penser que le nombre de canaux au niveau de la touffe ciliaire est relativement faible et suggère même la présence d'un seul canal par stéréocil $[8,9]$.

En l'absence de données histochimiques et morphologiques, la localisation possible des sites de transduction n'est suggérée que par des expériences indirectes qui, pour le moment, se contredisent. Lors de stimulations mécaniques, la mesure du changement local de potentiel extracellulaire au voisinage des cils des cellules ciliées du saccule chez le crapaud suggère que ces canaux sont localisés au sommet des cils [8].

Cette localisation indirecte des canaux de transduction est bien corrélée à la présence des liens apicaux interciliaires reliant le sommet des cils les plus courts aux cils adjacents plus longs. Selon la théorie d'Hudspeth [8], les liens interciliaires, décrits dans le paragraphe précédent (figure 1c), seraient directement reliés au canal de transduction et permettraient leurs ouverture et fermeture. Ces liens auraient des propriétés élastiques et seraient les éléments mécaniques qui provoquent l'ouverture des canaux (figure 2a) : la tension de ces liens augmenterait lors de la déflexion de la touffe ciliaire vers les cils les plus grands (vers le kinocil ou sa racine) et provoquerait l'ouverture du canal de transduction (figure 2a). Cependant ce modèle n'est pas vérifié dans les expériences d'Ohmori sur des cellules ciliées du poulet [10]. Ohmori utilise un indicateur fluorescent intracellulaire sensible au calcium (fura-2) afin de visualiser le passage des ions au travers des $\mathrm{m} / \mathrm{s} n^{\circ} 8$, vol. 6, octobre 90

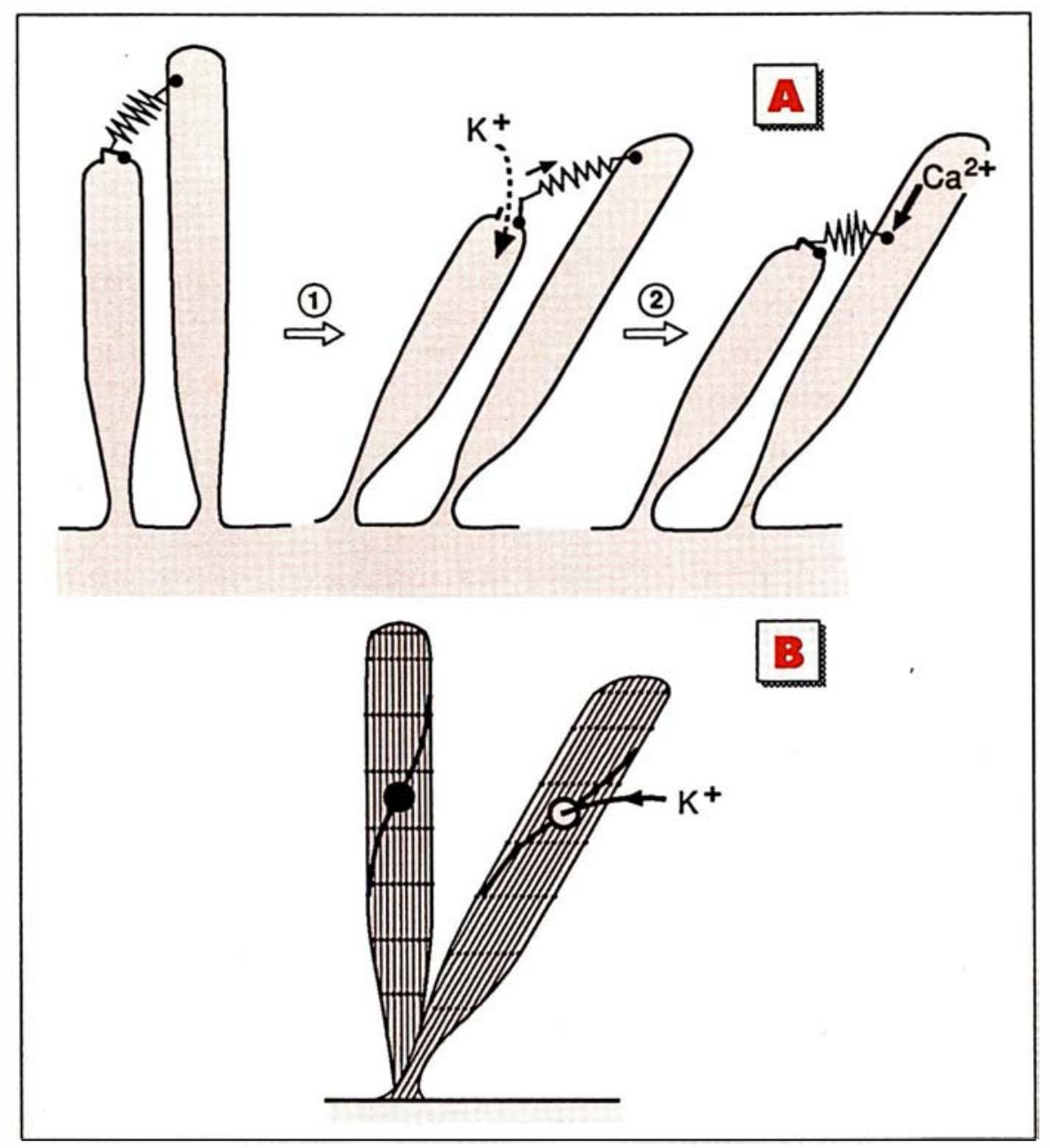

Figure 2. Deux modèles de fonctionnement des canaux de mécanotransduction. A : modèle proposé par Hudspeth et al. faisant intervenir les liens interciliaires apicaux dans les mécanismes d'activation des canaux de transduction (voir figure 1c). Ces liens relieraient le canal ionique situé à l'apex du cil le plus court au cil de la rangée adjacente. Lors de la stimulation mécanique provoquée par l'onde acoustique (1) la tension du lien de nature élastique augmente et provoque l'ouverture du canal qui laisse passer les ions $\mathrm{K}^{+}$ de l'endolymphe. Cette activation du canal de transduction sera à l'origine d'une dépolarisation cellulaire et de la libération des neurotransmetteurs. L'étape (2) schématise le phénomène d'adaptation de la transduction par le calcium. Le canal de transduction se referme alors que le cil est encore dans sa phase de stimulation. Cette adaptation correspondrait au glissement du lien le long du cil [18]. Ce glissement, dépendant du calcium, diminue la tension exercée sur le canal et provoque sa fermeture. B : modèle proposé par Sachs et al. identifiant les canaux de transduction des cellules ciliées aux canaux de transduction mécanique (stretch activated channels) que l'on trouve au niveau de nombreux types cellulaires telles les cellules musculaires. L'étirement ou stretch de la membrane latérale des stéréocils lors de leur déflexion provoquerait l'ouverture de ces canaux. 
canaux de transduction. Ses résultats suggèrent au contraire une localisation des canaux de transduction dans la région basale des stéréocils, probablement au point d'ancrage de ceux-ci dans la plaque cuticulaire [10].

La transduction mécano-électrique dans d'autres systèmes. Sachs et ses collaborateurs démontrèrent pour la première fois en 1984 l'existence de canaux ioniques activés par une force mécanique appliquée sur la membrane plasmique de cellules musculaires, les stretch-activated channels [11]. Depuis, l'existence de ce type de conductance ionique activée par une déformation de la membrane plasmique a été démontrée dans de très nombreux types cellulaires [12]. Il est très intéressant de noter que l'activité électrique des canaux de transduction des cellules ciliées sensorielles et des stretch-activated channels des cellules musculaires présentent de nombreuses homologies. Ils ont une conductance du même ordre et une absence de sélectivité cationique (ils peuvent laisser passer des ions très différents tels que le calcium ou le potassium). Ils ont en commun un même inhibiteur, l'antibiotique aminoglycosidique streptomycine $[12,13]$. Ces similitudes tendent à montrer qu'il s'agit peut-être de canaux ioniques identiques. Le fonctionnement de ce type de canal fait intervenir un cortex sous-membranaire, composé de filaments d'actine et de protéines telle la spectrine, associés mécaniquement au canal. Ces filaments d'actine existent en effet au niveau du stéréocil et Sachs propose un modèle simple particulièrement attractif pour le fonctionnement des canaux de transduction des cellules sensorielles [12]. Il n'est pas nécessaire, comme dans le modèle d'Hudspeth, de faire intervenir les liens apicaux interciliaires qui tirent le canal pour l'ouvrir. Le mouvement de la touffe ciliaire produirait un étirement ou stress membranaire au niveau de la partie médiane des cils, du fait de l'arrangement longitudinal des filaments d'actine intracellulaire. Cet étirement membranaire pourrait être à l'origine de l'ouverture d'un canal strech-activated (figure 2b).

Une meilleure connaissance du fonc- tionnement de ces canaux stretchactivated au niveau des cellules musculaires et leur caractérisation physique par la biologie moléculaire permettront très bientôt de les étudier au niveau des cellules ciliées. En fait ces canaux stretch-activated viennent très récemment d'être mis en évidence au niveau du corps cellulaire des cellules ciliées externes de la cochlée chez le cobaye [14]. Ils pourraient constituer des canaux de transduction mécano-électrique directe au niveau de la paroi latérale des cellules ciliées externes. L'expérience de Brundin et al. [15], décrivant une réponse des cellules in vitro lors de la stimulation acoustique très locale du mur latéral, renforcerait cette hypothèse.

Mécanismes de contrôle de la transduction mécano-électrique. Il existerait deux types de régulation, ou adaptation, de la mécanotransduction : d'une part, des processus de contrôle direct de l'activité des canaux de transduction eux-mêmes et, d'autre part, un contrôle des propriétés mécaniques des stéréocils. Ces mécanismes pourraient intervenir directement à travers une boucle de rétroaction intracellulaire rapide et indirectement par l'intermédiaire du système efférent, par exemple le système efférent médian innervant les cellules ciliées externes dans la cochlée [16].

L'adaptation de l'activité des canaux de transduction est exprimée par la diminution progressive du courant de transduction au cours d'une déflexion soutenue de la touffe ciliaire de cellules isolées in vitro [17]. Cette diminution du courant de transduction peut être interprétée comme une fermeture du canal alors que le cil est encore dans sa phase de stimulation. Ce phénomène est dépendant du calcium et correspondrait à une modification de la tension exercée par le lien, vraisemblablement élastique, attaché au canal de transduction [18]. Cette adaptation correspondrait au glissement du lien sur le cil adjacent du côté opposé au canal, entraînant ainsi une diminution progressive de la tension exercée sur celui-ci figure 2a). Ce glissement pourrait faire intervenir des molécules de myosine glissant sur les filaments d'actine
25. Nakamura T, Gold GH. A cyclic nucleotid-gated conductance in olfactory receptor cilia. Nature 1987 ; 325 : 442-4. 


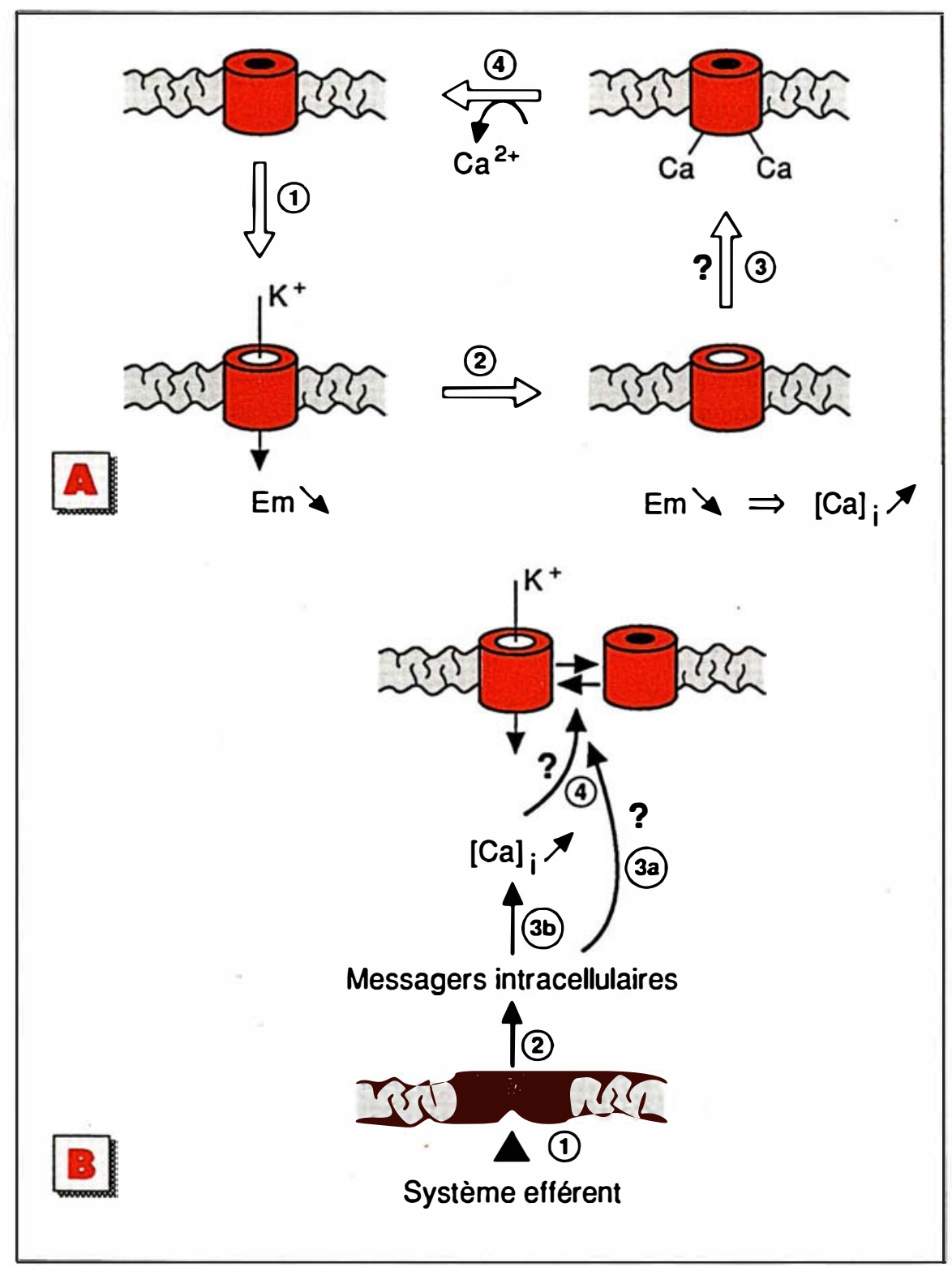

Figure 3. Hypothèses sur les étapes de contrôle de la mécanotransduction. A : autorégulation du canal de transduction : (1) la stimulation mécanique engendre l'ouverture du canal et conduit à une dépolarisation cellulaire (Em \); (2) Une élévation du calcium intracellulaire au voisinage du canal provient de l'entrée de Ca $2+$ via le canal lui-même ou de l'activation de canaux calcium dépendants du voltage; (3) Par analogie avec les stretch activated channels, le calcium diminuerait la conductance du canal de transduction des cellules ciliées; (4) Retour à l'étape initiale après contrôle de I'homéostasie calcique intracellulaire. B : contrôle par le système efférent: (1) Occupation du récepteur cellulaire (probablement muscarinique) par le ou les neurotransmetteurs du système efférent (probablement l'ACh) ; (2) Le récepteur cellulaire stimule la production de messagers secondaires (GMPc?, AMPc?, (P3?) ; (3) et (4) Ces messagers intracellulaires pourraient activer ou inhiber, par l'intermédiaire de kinases, les canaux de transduction (3a) ou pourraient être, dans le cas d'une production d'IP3, à l'origine d'une êlévation du calcium intracellulaire (3b) qui contrôlerait alors l'activité du canal (4).

$m / s n^{\circ} 8$, vol. 6, octobre 90 intraciliaire. Ce phénomène d'adaptation du courant ionique transducteur peut également s'intégrer dans le modèle des canaux stretch-activated de Sachs (figure 2b). Le calcium pourrait directement contrôler l'activité de ces canaux de transduction (figure $3 a$ ) et cela par homologie avec les canaux mécaniques de la cellule musculaire, dont la conductance est modifiée par le calcium [19]. En effet, les cellules ciliées externes possèdent des canaux calcium voltage-dépendants qui sont activés lors de la dépolarisation de la cellule suite à une stimulation acoustique [20, 21].

L'autre voie de contrôle de la mécanotransduction fait intervenir le système efférent. En effet, sa stimulation est capable de modifier la courbe d'accord (courbe de réponse en fonction de la fréquence) des cellules ciliées [22]. Des récepteurs muscariniques ayant été démontrés in vitro au niveau de cellules ciliées cochléaires du poulet [23], leur activation par l'innervation efférente provoquerait une augmentation du $\mathrm{Ca}^{2}+$ intracellulaire qui pourrait être à l'origine de la régulation des canaux de transduction (figure $3 b$ ). Il est intéressant de noter dans ce contexte qu'il a été récemment montré dans la cochlée des mammiferes que l'acétylcholine, par l'intermédiaire d'un récepteur caractérisé comme muscarinique de type M3, était capable de stimuler la boucle de synthèse des phospho-inositides à l'origine de la production d'IP3 et d'IP4 [24], molécules qui jouent le rôle de messagers responsables des variations du calcium intracellulaire dans de nombreux autres types cellulaires.

Bien qu'on ne dispose pour l'instant d'aucune preuve en ce qui concerne les cellules ciliées, un autre type de contrôle ou de régulation de la mécanotransduction pourrait faire intervenir des nucléotides cycliques tels que l'AMPc ou le GMPc. Ces molécules sont en effet capables de régler, directement ou indirectement (stimulation de protéine kinase phosphorilant le canal), l'activité de canaux ioniques dans la transduction olfactive et visuelle, par exemple [1,25]. Cette modulation ou adaptation par de tels messagers intracellulaires pourrait aussi exister au niveau des cellules ciliées et provenir d'une activation du système efférent. 


\section{RÉFÉRENCES}

26. Orman S, Flock $\AA$. Active control of sensory hair mechanics implied by susceptibility to media that induce contraction in muscle. Hear Res 1983; 11 : 261-6.

27. Kossl M, Richardson GP, Russel IJ. Stereocilia bundle stiffness : effects of neomycin sulphate, A23187 and concanavalin A. Hearing Res 1990; 44 : 217-30.

28. Aran JM. L'amplificateur cochléaire. médecine/sciences $1990 ; 6: 740-2$.

29. Lim DJ. Cochlear anatomy related to cochlear micromechanics. A review. $J$ Acoust Soc Am 1980 ; 67 : 1686-95.

30. Khanna SM, Leonard DGB. Basilar membrane tuning in the cat cochlea. Science $1982 ; 215$ : 305-6.

31. Gummer AW, Smolders JWT, Klinke $\mathrm{R}$. Basilar membrane motion in the pigeon measured with the Mossbauer technique. Hearing Res 1987; 29 : 63-92.

32. Fettiplace R. Electrical tuning of hair cells in the inner ear. Trends in Neurose $1987 ; 10: 421-5$

33. Brownell WE, Bader CR, Bertrand D, de Ribeaupierre Y. Evoked mechanical responses of isolated cochlear hair cells. Science 1985 ; 227 : 194-6.

34. Ashmore JF. A fast motile response in guinea pig outer hair cells : the cellular basis of the cochlear amplifier. $J$ Physiol Lond 1987 ; 388 : 323-47.

35. Dulon D, Zajic G, Schacht J. Increasing intracellular free calcium induces circumferential contractions in isolated cochlear outer hair cells. $J$ Neurosci $1990 ; 10$ : 1388-97.

36. Holley MC, Ashmore JF. A cytoskeletal spring in cochlear outer hair cells. Nature $1988 ; 335$ : 635-7.

37. Plinkert PK, Gitter AH, Zimmermann U, Kirchner T, Tzartos S, Zenner H P. Visualization and functional testing of acetylcholine receptor-like molecules in cochlear outer hair cells. Hearing Res 1990 ; 44 : 25-34.

38. Didier A, Decory L, Cazals Y. Evidence for potassium-induced motility in type

I vestibular hair cells in the guinea pig.
Enfin, un autre type de contrôle de la mécanotransduction pourrait reposer directement sur la modulation des propriétés mécaniques des stéréocils, ceci par l'intermédiaire des protéines contractiles effectivement présentes dans la plaque cuticulaire et les cils. L'élément régulateur pourrait là aussi être le calcium. En effet, le traitement des touffes ciliaires des cellules ciliées in vitro par un ionophore calcique, permettant d'élever la concentration intracellulaire du calcium, produit une augmentation de la rigidité des cils [26, 27] qui devrait se traduire par une modification de la sensibilité mécanique du système. Là aussi la boucle de contrôle pourrait être directe et rapide, ou plus lente avec l'intervention du système efférent.

\section{Base cellulaire de I'analyse fréquentielle par le récepteur auditif}

Comme il est rappelé par ailleurs (éditorial de J.M. Aran dans ce numéro [28] et article de R. Pujol [16]), la très grande sélectivité fréquentielle auditive, telle qu'elle peut être caractérisée par la courbe d'accord d'une fibre du nerf auditif, apparaît dès le niveau de la réponse mécanique de la membrane basilaire chez les mammifères, et est notamment très dépendante de l'état physiologique des cellules ciliées externes. Quels sont les mécanismes cellulaires qui assurent cette fonction? On sait, depuis Békésy, que l'analyse du signal acoustique repose sur les propriétés purement mécaniques de la membrane basilaire. Parallèlement à l'évolution des caractéristiques physiques de cette membrane de la base vers l'apex (masse, épaisseur, raideur) qui lui permet de réaliser une analyse mécanique grossière des fréquences, des aigues aux graves, il existe des changements progressifs des caractéristiques morphologiques des cellules et des touffes ciliaires elles-mêmes [29]. Il s'agit principalement de la longueur des cellules et des cils qui va croissante de la base vers l'apex de la cochlée. Ainsi la hauteur des touffes ciliaires passe de $1 \mu \mathrm{m}$ à la base à $6 \mu \mathrm{m}$ environ à l'apex. Il existe donc une relation précise entre les dimensions de la membrane basilaire, celles des cellules sensorielles et la fréquence caractéristique, mais ces seules données physiques ne paraissent pas suffisantes pour expliquer la grande sélectivité des réponses observées, et il apparaît que celle-ci repose surtout sur les propriétés physiologiques intrinsèques des cellules ciliées ellesmêmes, différemment exprimées chez les vertébrés inférieurs et les mammifères.

La sélectivité fréquentielle chez les amphibiens, les reptiles et les oiseaux repose sur les propriétés électriques intrinsèques de chaque cellule ciliée. Si la très grande sélectivité fréquentielle de la cochlée des mammifères s'observe dès le mouvement de la membrane basilaire [30], il ne semble pas que ce soit le cas chez les vertébrés inférieurs ou les oiseaux, où la courbe d'accord mécanique est moins affinée que celle des fibres nerveuses [31]. En revanche, la réponse la plus sélective apparaît au niveau de la résonance électrique du potentiel récepteur des cellules sensorielles [32].

En effet, les cellules ciliées des vertébrés inférieurs ont des propriétés membranaires très particulières : leur potentiel de membrane est capable de résonner électriquement à une fréquence très précise. Ainsi, l'injection d'un courant dans la cellule est suivie, non pas d'un retour exponentiel du potentiel membranaire à son niveau d'équilibre, mais d'une oscillation amortie de celui-ci à une fréquence bien définie (figure 4a). De même, lors d'une stimulation acoustique brève (clic), la cellule produit un potentiel récepteur qui oscille à la même fréquence (figure 4b). L'amplitude du potentiel récepteur est maximale lorsque la fréquence de stimulation acoustique coïncide avec la fréquence de résonance électrique de la cellule. De ce fait, chaque cellule le long de la cochlée est à l'origine d'une excitation maximale de la fibre nerveuse postsynaptique pour une fréquence acoustique bien précise. Cette propriété de résonance électrique et mécano-électrique des cellules ciliées peut être expliquée par la mise en jeu combinée de courants opposés de canaux potassium et de canaux calcium [32]. Cette fréquence 
de résonance du potentiel récepteur augmente graduellement de la base à l'apex de la cochlée. Les raisons de cet arrangement tonotopique des propriétés de résonance électrique cellulaire le long de la cochlée sont encore mal connues, mais il pourrait s'expliquer par une augmentation progressive du nombre des canaux $\mathrm{K}+$ et $\mathrm{Ca} 2+$ de chaque cellule ou par des différences de cinétique de ces canaux. Cet arrangement tonotopique est à rapprocher également des variations progressives de la taille des cellules et des touffes ciliaires.

Cette propriété de résonance amortie du potentiel récepteur n'a pas été observée au niveau des cellules ciliées isolées de la cochlée des mammifères, où la sélectivité fréquentielle ferait intervenir un processus cellulaire différent.

La sélectivité fréquentielle des mammifères repose sur les propriétés mécaniques actives des cellules ciliées externes. Comme il est amplement décrit par ailleurs [16, 28], les cellules ciliées internes dans la cochlée, qui sont pratiquement les seules branchées sur le nerf auditif, sont peu sensibles et elles ne peuvent transmettre un message complet au nerf que parce qu'elles reçoivent une vibration mécanique amplifiée sélectivement en fonction de la fréquence - par les cellules ciliées externes. Celles-ci sont, en effet, extrêmement sensibles sur le plan mécanique et réalisent, dès l'arrivée de l'onde acoustique, une transduction mécano-électrique pour des mouvements de la membrane basilaire dont l'amplitude est de l'ordre de grandeur du diamètre de l'atome d'hydrogène. Ensuite, à travers des processus intracellulaires, elles effectuent une amplification et une transduction électro-mécanique en sens inverse. Cet ensemble de processus inclut des mécanismes de contrôle, biochimiques et in fine mécaniques, intrinsèques et/ou placés sous l'influence du système nerveux central.

En effet, il a été démontré, à l'occasion d'observations sur cellules externes isolées in vitro, que celles-ci étaient capables d'osciller mécaniquement à des fréquences acoustiques (plusieurs $\mathrm{kHz}$ ) sous stimulation élec- trique $[33,34]$. Ce type de mobilité est caractéristique des cellules ciliées externes et n'existe pas dans les cellules ciliées internes ou les cellules vestibulaires. Cette mobilité paraît essentiellement fonction du potentiel membranaire de la cellule, elle ne fait pas intervenir l'hydrolyse de molécules chimiques énergétiques telles que l'ATP et elle est indépendante du calcium intracellulaire [34]. Les bases biophysiques de cette mobilité dépendante du voltage restent encore inconnues. Il pourrait s'agir de processus électro-osmotiques au niveau de la paroi latérale de ces cellules [33]. Comme il a été mentionné plus haut, il est intéressant de noter que lors de la stimulation électrique on n'observe pas, à l'inverse de ce qui se passe dans les cellules des vertébrés inférieurs, de résonance du potentiel récepteur. Lors de la stimulation électrique, on ne note pas non plus de fréquence particulière pour laquelle la réponse mécanique de cette cellule serait maximale. Néanmoins on peut penser, mais il n'existe aucune preuve directe à ce jour, que la déflexion des stéréocils des cellules ciliées externes produit un potentiel récepteur qui serait à l'origine des oscillations mécaniques en retour de leur corps cellulaire, oscillations qui seraient alors transmises aux touffes ciliaires des cellules ciliées internes (figure 5A). Ce sont ces oscillations qu'on observerait lors de la mesure de la vibration de la membrane basilaire. Elles seraient égale-

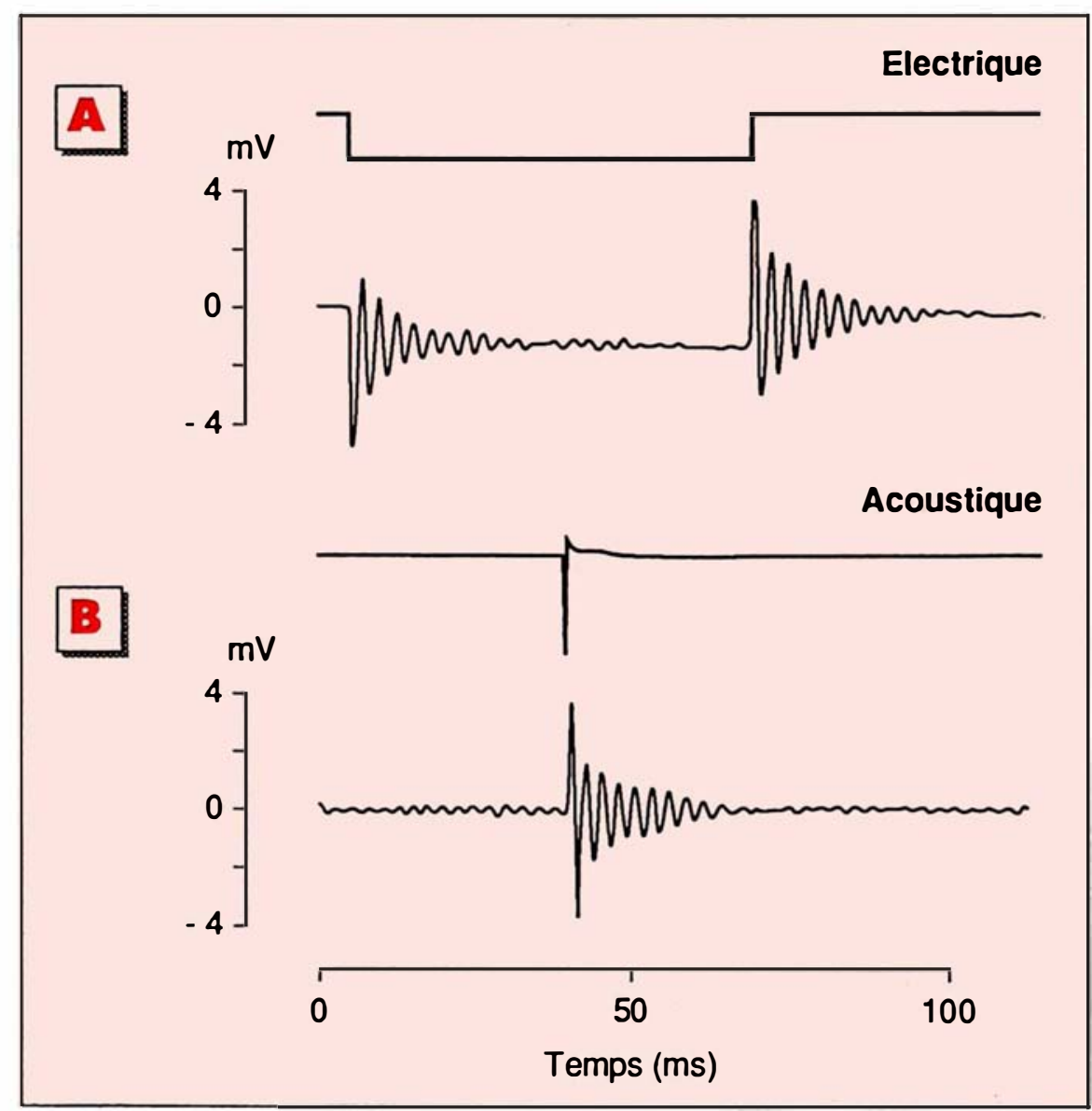

Figure 4. Propriété de résonance électrique des cellules ciliées des vertébrés inférieurs (d'après Fettiplace [32]). Une stimulation électrique par l'intermédiaire $d^{\prime}$ une électrode intracellulaire $A$ ou une stimulation acoustique brève (clic) $B$ produisent au niveau d'une même cellule ciliée une oscillation amortie du potentiel membranaire. Noter que la fréquence d'oscillation pour les deux types de stimulation est identique. Cette fréquence est caractéristique de chaque cellule ciliée le long de la cochlée (ici $370 \mathrm{~Hz}$ ) et correspond à la fréquence acoustique à laquelle la cellule est le plus sensible, c'est-à-dire la fréquence à laquelle la cellule produit un potentiel récepteur maximal. 
ment à l'origine des émissions acoustiques enregistrées devant le tympan [16].

Par ailleurs, une contraction ou élongation lente de la cellule ciliée externe in vitro a été observée lors de stimulations acoustiques appliquées au niveau de la paroi latérale [15]. Cette déformation se maintient pendant toute la durée de la stimulation et, fait remarquable, son amplitude

Figure 5. Hypothèses sur les bases cellulaires et moléculaires de la transduction mécano-électrique cochléaire chez les mammifères. A : I'amplificateur cochléaire : une stimulation acoustique de faible intensité provoque la déflexion des cils des cellules ciliées externes (CCE) qui sera à l'origine d'un potentiel récepteur (Em) (1a) . La dépolarisation cellulaire active a son tour un courant sortant de $K+$ permettant une repolarisation (2a). Ces variations du potentiel membranaire (2a-3a) sont à l'origine d'une oscillation mécanique des CEE (40) qui stimulerait les cils des cellules ciliées internes (CCI). Les CCl transmettent alors l'information acoustique au nerf auditif (5a). Une boucle de contrôle des oscillations mécaniques des CCE serait mise en jeu à un certain seuil de dépolarisation cellulaire 6a). Des canaux $\mathrm{Ca}^{2}+$ dépendants du voltage produiraient une élévation du calcium intracellulaire qui serait à l'origine de contractions de type acto-myosine du cortex membranaire (7a) dépendantes de la calmoduline ( $\mathrm{CaM})$. Ces contractions permettraient le contrôle lamortissement ?) des oscillations mécaniques dépendantes du voltage des CCE (8). $B$ : intervention du système efférent dans le contrôle des oscillations mécaniques rapides dépendantes du voltage des CCE (8). La liaison des neurotransmetteurs, dont I'ACh lacétylcholine) est le plus probable, au récepteur muscarinique cellulaire (16) stimulerait la production de messagers secondaires (GMPc?, IP3?) (IP3 = inositol 1, 4, 5, triphosphatel (2b). Ces messagers secondaires produiraient une élévation du calcium intracellulaire (3b) et en conséquence une contraction du cortex acto-myosinique sous-membranaire (4b). Ces seconds messagers pourraient aussi être à l'origine du contrôle de l'activité des canaux ioniques membranaires (5b) et 6b. . Aux fortes intensités, l'ensemble des phénomènes liés aux CCE saturent et la vibration s'adresse directe- est maximale pour une stimulation à la fréquence qui correspond au point d'origine de la cellule sur la membrane basilaire. La courbe de réponse en fonction de la fréquence est comparable à la courbe de sélectivité cochléaire, et on a ici pour la première fois la démonstration d'une sélectivité fréquentielle intrinsèque de la cellule ciliée externe chez le mammifere, puisqu'on l'observe sur la cellule isolée, in vitro. Les mécanismes biochimiques cellulaires responsables de ce type de mouvement ne sont pas encore définis. Cependant, des modifications de forme du corps cellulaire des cellules ciliées externes, apparentées à des contractions de type musculaire, ont été observées lors de l'élévation du $\mathrm{Ca}^{2}+$ intracellulaire [35]. Ces contractions représenteraient la réponse mécanique

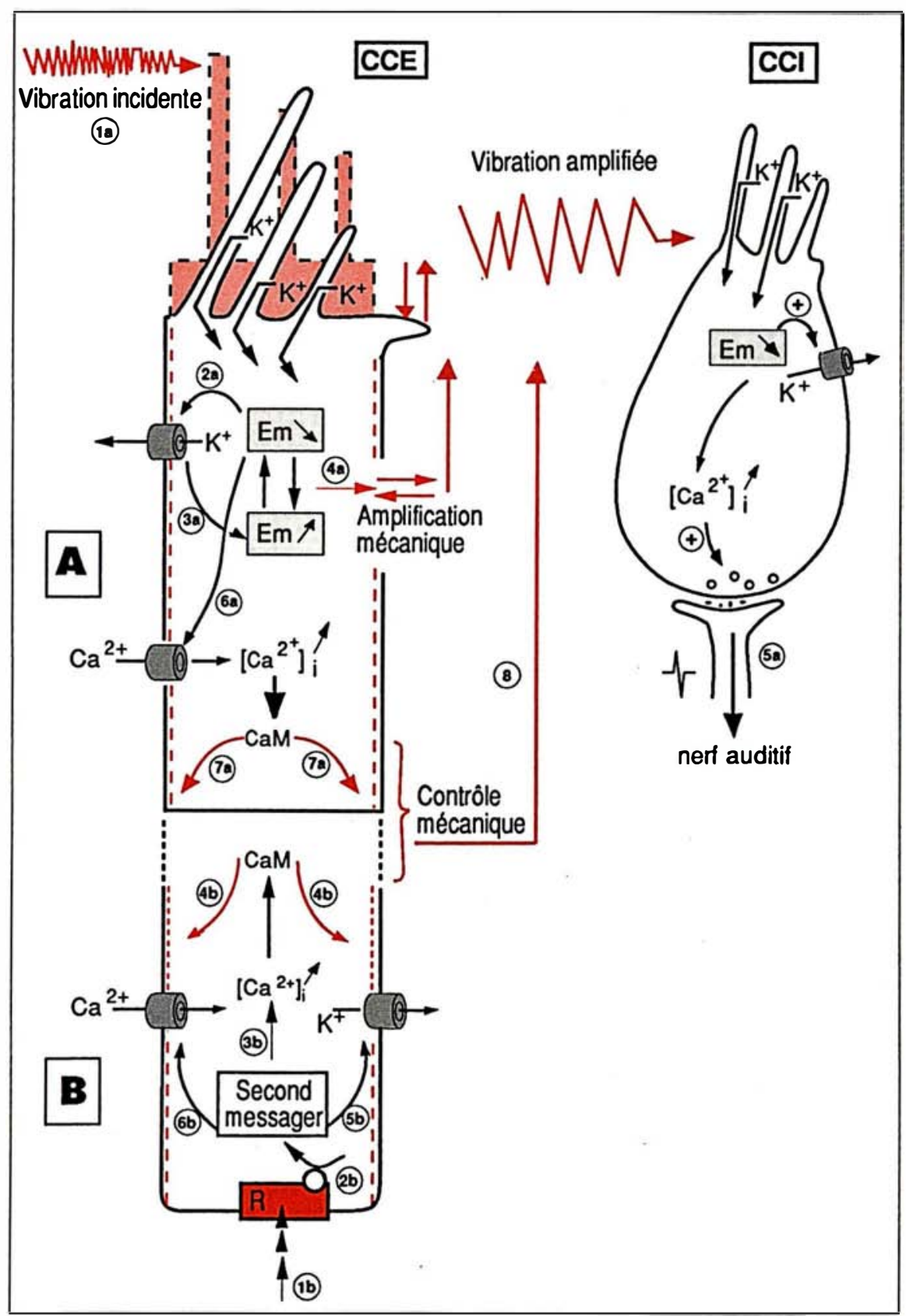


d'un cortex circonférentiel sousmembranaire [36] qui modifie la tonicité cellulaire. La calmoduline, protéine liant le calcium et intervenant dans les mécanismes de contraction des cellules musculaires et non musculaires, semble impliquée dans ce type de contraction des cellules ciliées externes [35] (figure 5b). Quel est le stimulus physiologique et le rôle de ces contractions dépendantes du calcium ? Une élévation du calcium intracellulaire peut provenir d'une libération de celui-ci à partir de magasins intracellulaires ou de l'activation de canaux membranaires dépendants du voltage présents au niveau de ces cellules [21] (figure 5a). Ces deux mécanismes pourraient effectivement être mis en jeu lors de la stimulation acoustique. L'acétylcholine, neurotransmetteur probable du système efférent, par analogie avec le système musculaire, pourrait être à l'origine de contractions dépendantes du calcium, par l'intermédiaire de récepteurs muscariniques couplés à une mobilisation du calcium intracellulaire (figure 5b). Cependant, dans des expériences in vitro sur cellules ciliées externes isolées, on n'a encore jamais observé, en présence d'acétylcholine, d'élévation du calcium intracellulaire [35] et seule une publication récente, à côté de nombreux résultats négatifs, rapporte une contraction induite [37]. Il se pourrait que l'acétylcholine fût simplement à l'origine de la production de messagers intracellulaires de type IP3, IP4, AMPc ou GMPc ayant pour rôle la régulation de l'activité des canaux ioniques membranaires de transduction, ou des canaux calcium ou potassium (figure 5b). Le rôle physiologique de ces contractions dépendantes du calcium reste encore hypothétique, mais on peut penser qu'elles jouent le rôle de rétrocontrôle, soit des oscillations mécaniques actives dépendantes du voltage de ces cellules, soit de leur amortissement, ou encore modifient, par exemple, leur sensibilité mécanique en fonction de la fréquence. Ce contrôle pourrait donc aussi bien s'exprimer par une boucle rétroactive intracellulaire rapide (figure 5a) que par une boucle rétroactive lente impliquant le système efférent (figure $5 b)$. Cette contraction dépendante du $\mathrm{m} / \mathrm{s} n^{\circ} 8$, vol. 6 , octobre 90 calcium contrôlerait la tonicité cellulaire des cellules ciliées externes et permettrait en conséquence de régler les propriétés mécaniques de la membrane basilaire.

\section{Conclusion}

Le temps de réponse extrêmement rapide des cellules ciliées sensorielles de l'oreille interne, de l'ordre de la microseconde dans la cochlée, est l'argument le plus percutant pour dire que la transduction mécanoélectrique est un phénomène direct qui ne fait pas intervenir de messagers intracellulaires comme dans la transduction photo-électrique ou chimique. Il y a ici un couplage direct entre la stimulation mécanique des stéréocils et l'ouverture de canaux ioniques à l'origine de la réponse électrique cellulaire. Même si l'on entrevoit parfaitement comment la transduction mécano-sensorielle par les cellules ciliées, notamment les cellules ciliées internes de la cochlée, se réalise dans ses grandes étapes qui sont le mouvement des cils, l'ouverture de canaux ioniques, la dépolarisation cellulaire et enfin la libération du neurotransmetteur (le glutamate) qui ira exciter les fibres afférentes du nerf, on connaît encore mal ces étapes dans leur détail et en particulier du point de vue biochimique et moléculaire.

La localisation précise de ces canaux ioniques mécano-récepteurs au niveau des stéréocils ainsi que les mécanismes de leur ouverture sont encore l'objet d'avis controversés. Ces canaux se situent-ils au point d'ancrage des cils dans la plaque cuticulaire ou à l'apex des cils ? Leur ouverture est-elle déclenchée par la traction d'un lien élastique interciliaire ou provient-elle simplement de l'étirement de la membrane plasmique?

La régulation de la mécanotransduction, en particulier par le système efférent, est aussi un point particulièrement important et fait l'objet de nombreuses recherches : si le glutamate est bien reconnu comme étant le neurotransmetteur afférent à l'origine de la transmission de l'information vers les centres nerveux, de nombreuses inconnues demeurent en ce qui concerne les neurotransmet- teurs impliqués dans ces mécanismes de régulation par le système nerveux efférent, et notamment la nature des messagers intracellulaires qui interviennent.

$\mathrm{Si}$ ces mécanismes primaires de la transduction mécano-électrique sont vraisemblablement les mêmes dans les différents types cellulaires, cellules ciliées internes et externes dans la cochlée, cellules de type I et de type II dans les récepteurs vestibulaires, les sensibilités respectives de ces cellules sont différentes, fonction de leur environnement, notamment sur le plan mécanique, et de leurs propriétés intrinsèques.

Ainsi, la mise en évidence de processus mécaniques actifs dans les cellules ciliées externes de la cochlée, qui sont à la base d'une amplification sélective des vibrations acoustiques, constitue véritablement l'avancée récente la plus importante dans ce domaine. Il est possible que certains mécanismes contractiles existent aussi dans d'autres types de cellules sensorielles de l'oreille interne. Ainsi des mouvements de cellules vestibulaires viennent récemment d'être décrits [38]. La compréhension des mécanismes cellulaires et moléculaires impliqués dans ces phénomènes constitue sans nul doute le défi le plus important à relever dans les prochaines années

\section{Summary}

Molecular and cellular basis of mechano-electrical transduction in the inner ear

The vertebrate cochlea has the dual function of receiving and analyzing complex acoustic stimuli, particularly in separating out their frequency components, and of encoding them into a sequence of impulses distributed within the fibers of the auditory nerve. This is achieved through the action of sensory hair cells.

During the acoustical stimulation, mechanical forces displace the hair bundle of individual hair cells. This motion modifies the gating state of mechano-sensitive transduction channels which leads to the electrical response of the cell. 


\section{Summary (suite)}

Although the molecular nature of such channels and the precise mechanisms of gating remain to be defined, these channels appear to be operated via calciumdependent elastic components in the hair bundle.

From then on, different intracellular events take place which realize the sound processing and the sensory-neural excitation. Although the typical sensoryneural transduction process is rather clearly understood, the intracellular processes and the cellular interactions performing the analysis of sound are extremely complex and appear to differ significantly between lower vertebrates and mammals.

In lower vertebrates the frequency selectivity is based on individual mechanical and electrical properties of the hair cells. They behave like electrical resonators, developing a maximal receptor potential which oscillates at a specific frequency. In mammals, in addition to the graded mechanical properties of the basilar membrane along the cochlea, responsible for a gross frequency analysis, a very sensitive and frequency-selective acoustic amplification is achieved through active mechanical processes by the outer hair cells. These cells, which possess contractile structures, have been demonstrated in vitro to generate tuned motile responses at specific acoustic frequencies, which would explain the highly tuned mechanical response of the basilar membrane recorded in vivo. It is assumed that the locally amplified and tuned vibration addresses the inner hair cells which in turn transduce this vibration into a chemical excitation of the auditory nerve. This mechanical selective amplification by the outer hair cells would be controlled via an efferent, cholinergic, innervation which is supposed to act upon another contractile $\mathrm{Ca} 2+/ \mathrm{ATP}$ dependent mechanism.

\section{TIRÉS A PART}

\section{APPEL D'OFFRES INSERM 1990}

Postes d'accueil dans le domaine de la Santé

Dans le cadre de l'inflexion de la politique en faveur du développement de la recherche en santé et plus spécifiquement en santé publique, I'Inserm engage un programme de formation à et par la recherche dans ces domaines et envisage de leur réserver dès 1991 au moins six postes d'accueil destinés à permettre à leurs bénéficiaires de se consacrer à plein temps à la recherche pendant une période de un à trois ans.

Les thèmes et disciplines concernés sont tous ceux qui intéressent la santé et en particulier la santé publique, notamment :

- au rang des thèmes : l'évaluation des techniques et des pratiques médicales et des systèmes de santé : la prévention; la prise en charge des états de dépendance :

- au rang des disciplines : l'épidémiologie, la sociologie, l'économie, et, de façon plus générale, les disciplines relevant des sciences de l'homme et de la société et concernant la santé.

Les postes seront attribués à des jeunes chercheurs en cours de formation, d'origine médicale ou non médicale, titulaires d'un DEA ou d'une formation jugée équivalente, et, pour les candidats non médecins, d'au moins un an d'activité au-delà du DEA dans un domaine relevant des indications précédentes.

La durée du contrat sera de un an, renouvelable deux fois. Le lieu d'accueil sera obligatoirement un laboratoire public spécialisé dans le domaine de la santé. L'accueil dans les laboratoires ayant une expérience reconnue en matière de formation sera privilégié. Les bénéficiaires pourront effectuer un stage à l'étranger au cours de la deuxième ou de la troisième année de leur accueil. Les modalités de rémunération seront déterminées par référence à celles du corps des chargés de recherche de l'Inserm.

La sélection des candidats sera effectuée fin janvier 1991 par une commission ad hoc présidée par un membre du Conseil scientifique de I'Inserm et comprenant quatre représentants de la commission scientifique spécialisée $n^{\circ} 8$ (Épidémiologie, santé publique, sciences sociales, environnement, toxicomanie), un représentant des quatre intercommissions actuellement en fonction ("Vieillissement", " Handicap ", "Évaluation Médicale ", "Prévention ") et six experts nommés.

La sélection sera fondée sur l'examen du dossier des candidats et de leur audition par une sous-commission issue de la commission ou par la commission elle-même. Les dossiers comprendront notamment l'exposé des titres et travaux du candidat, celui des motivations qui l'orientent vers la recherche en santé et celui de son projet de recherche. Ils comprendront également toutes informations utiles sur le laboratoire d'accueil et sur ses activités spécifiques dans le domaine choisi par le candidat, ainsi que le nom du ou des chercheurs plus spécifiquement chargés de contribuer à sa formation.

\section{Date limite de dépôt ou d'envoi des dossiers 14 DÉCEMBRE 1990 \\ (le cachet de la poste faisant foi)}

Les formulaires de demande de postes $d$ 'accueil en santé sont disponibles sur demande écrite au bureau des concours chercheurs, Inserm, 101, rue de Tolbiac (3e étage porte 337), 75654 Paris Cedex 13. Tél. : 45.84.14.41 (postes 4361 à 4365). 